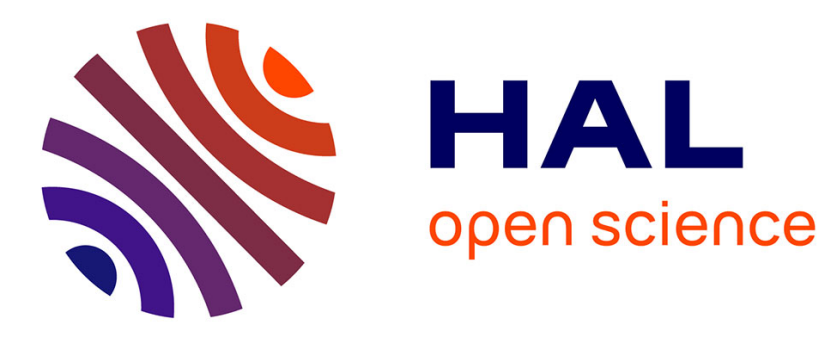

\title{
MIMO Hardware Simulator Design for Outdoor Time-Varying Heterogeneous Channels
}

\author{
Bachir Habib, Gheorghe Zaharia, Ghais El Zein
}

\section{To cite this version:}

Bachir Habib, Gheorghe Zaharia, Ghais El Zein. MIMO Hardware Simulator Design for Outdoor Time-Varying Heterogeneous Channels. International Symposium on Signals, Circuits and Signals, Jul 2013, Iasi, Romania. pp.1-4. hal-00871071

\section{HAL Id: hal-00871071 \\ https://hal.science/hal-00871071}

Submitted on 8 Oct 2013

HAL is a multi-disciplinary open access archive for the deposit and dissemination of scientific research documents, whether they are published or not. The documents may come from teaching and research institutions in France or abroad, or from public or private research centers.
L'archive ouverte pluridisciplinaire $\mathbf{H A L}$, est destinée au dépôt et à la diffusion de documents scientifiques de niveau recherche, publiés ou non, émanant des établissements d'enseignement et de recherche français ou étrangers, des laboratoires publics ou privés. 


\title{
MIMO Hardware Simulator Design for Outdoor Time-Varying Heterogeneous Channels
}

\author{
Bachir Habib, Gheorghe Zaharia, Ghaïs El Zein \\ Institute of Electronics and Telecommunications of Rennes (IETR), UMR CNRS 6164, Rennes, France \\ bachir.habib@insa-rennes.fr
}

\begin{abstract}
This paper presents a hardware simulator of MultipleInput Multiple-Output (MIMO) propagation channels. The hardware simulator reproduces a desired radio channel and makes it possible to test "on table" different MIMO systems. A specific architecture of the digital block of the simulator is presented to characterize an outdoor scenario for Long Term Evolution (LTE) systems. An algorithm is introduced to switch between the impulse responses and to control the time variation of the delays. The new architecture is designed on a Xilinx Virtex-IV Field Programmable Gate Array (FPGA). Its accuracy, occupation on the FPGA and latency are analyzed.
\end{abstract}

Keywords-Hardware simulator; MIMO channels; heterogeneous environments; LTE; FPGA

\section{INTRODUCTION}

MIMO techniques improve the capacity and the performance of wireless communication systems. Several studies published recently present systems that reach a MIMO order of $8 \times 8$ and higher [1]. This is made possible by advances at all levels of the simulator platforms [2]. With continuing increase of the Field Programmable Gate Array (FPGA) capacity, entire baseband systems can be mapped onto faster FPGAs for more efficient prototyping and testing [3]. Some MIMO hardware simulators are proposed by industrial companies like Spirent [4], Azimuth (ACE), Elektrobit (Propsim F8) [5], but they are quite expensive and they do not cover all types of environments.

The channel models can be obtained from standard models, as the TGn 802.11n [6] and the LTE models [7], or from measurements conducted with the MIMO channel sounder designed and realized at IETR [8]. In the MIMO context, little experimental results have been obtained regarding timevariations, partly due to several limitations of the channel sounding equipment [9]. However, theoretical models of timevarying channels can be obtained using Rayleigh fading [10]. At IETR, several architectures of the digital block of a hardware simulator have been studied [11]. Typically, radio propagation channels are simulated using finite impulse response (FIR) filters, as in [11, 12]. The Fast Fourier Transform (FFT) modules with algebraic product can also be used, as in [13].

The frequency architecture considered in [13] operates correctly for signals not exceeding the FFT size. Thus, new frequency architecture avoiding this limitation has been presented and tested in [14]. However, [15] and [16] show that the time domain architecture is better in terms of occupation on FPGA, output error and latency. Therefore, in this paper, only the time domain architecture is considered.
The main contributions of the paper are:

- Tests have been made for indoor [15] and outdoor [16] fixed environments using standard channel models. In this paper, an algorithm is proposed to switch between several outdoor environments and make it possible to simulate heterogeneous radio propagation channels.

- In order to simulate a time-varying channel, a Rayleigh fading method is used. Moreover, the delays variation of the impulse response taps depends on the Doppler spread and the proposed environment. Therefore, to decrease the number of multipliers on the FPGA, a solution is proposed to control the change of delays in the architecture.

The rest of this paper is organized as follows. Section II presents the channel characteristics. Section III describes the architecture and its hardware implementation. In Section IV, the accuracy of the architecture is analyzed. Lastly, Section V gives some concluding remarks and prospects.

\section{CHANNEL DESCRIPTION}

\section{A. Proposed Scenario}

The scenario covers outdoor environments at different environmental speeds. It considers the movements of a person from an environment to another using LTE signal with a sampling frequency $\left(f_{s}\right)$ of $50 \mathrm{MHz}$, a sampling period $\left(T_{s}\right)$ of 20 ns, a bandwidth $(B)$ of $20 \mathrm{MHz}$ and a central frequency $\left(f_{c}\right)$ of $1.8 \mathrm{GHz}$. This person driving his vehicle, moves from a highway to an urban environment. For this scenario, the LTE Extended Vehicular A (EVA) model and the LTE Extended Typical Urban (ETU) model are used for this scenario. The relative power of each tap of the impulse response for the considered LTE channel models are presented [7] by taking the Line-Of-Sight (LOS) path as reference. Fig. 1 and Table I describe the proposed scenario.

$v$ is the mean environmental speed, $f_{\text {ref }}$ is the refresh frequency between two successive MIMO profiles, $t$ is the duration of movements in the considered environment, $d$ is the traveled distance and $N_{p}=t \times f_{\text {ref }}$ is the number of profiles in each environment.

$f_{d}$ is the Doppler frequency and it is equal to:

$$
f_{d}=\frac{f_{c} \cdot v}{c}
$$

where $c$ is the celerity. $f_{\text {ref }}$ is chosen greater than $2 f_{d}$ to respect the Nyquist-Shannon sampling theorem. 


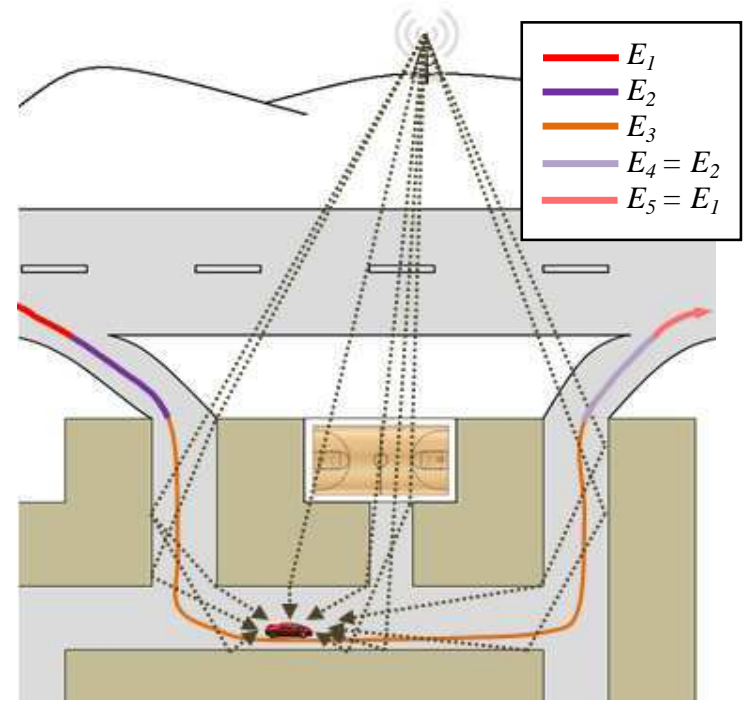

Figure 1. Proposed scenario.

TABLE I. SCENARIO DESCRIPTION

\begin{tabular}{|c|c|c|c|c|c|c|c|}
\hline Environnent & Model & $\begin{array}{c}v \\
(\mathrm{~km} / \mathrm{h})\end{array}$ & $\begin{array}{c}f_{d} \\
(\mathrm{~Hz})\end{array}$ & $\begin{array}{c}f_{\text {ref }} \\
(\mathrm{Hz})\end{array}$ & $\begin{array}{c}t \\
(\mathrm{~s})\end{array}$ & $\begin{array}{c}d \\
(\mathrm{~km})\end{array}$ & $N_{p}$ \\
\hline $\boldsymbol{E}_{\boldsymbol{1}}$ & EVA & 120 & 200 & 450 & 300 & 10 & 135,000 \\
\hline $\boldsymbol{E}_{\boldsymbol{2}}$ & EVA & 80 & 133 & 300 & 36 & 0.8 & 10,800 \\
\hline $\boldsymbol{E}_{\boldsymbol{3}}$ & ETU & 40 & 66 & 150 & 18 & 0.2 & 2,700 \\
\hline
\end{tabular}

The switch between the environments must be made in continuous manner. Between $E_{1}$ and $E_{2}$ for example, the vehicle speed decreases from $120 \mathrm{~km} / \mathrm{h}$ to $80 \mathrm{~km} / \mathrm{h}$. Thus, we consider a mean environmental speed for each environment.

\section{B. Algorithm Design to Switch between Environments}

To switch from 9 taps $\left(E_{2}\right)$ with a maximum excess delay of $2510 \mathrm{~ns}$ to 9 taps $\left(E_{3}\right)$ with a maximum delay of $5000 \mathrm{~ns}$, an algorithm is proposed. Two parameters are considered: the delay of the taps and their relative powers:

1) The number of taps is the same for $E_{2}$ and $E_{3}$. Therefore, the delays of $E_{3}$ are increased by $T_{s}=20 \mathrm{~ns}$ each $1 / f_{\text {ref }}$ to achieve the same delay vector of $E_{2}$. This procedure takes $(5000-2510) /\left(T_{s} \times f_{\text {ref }}\right)=125 / f_{\text {ref }}$ seconds to be completed.

2) To pass from the last $h_{11}$ profile in $E_{2}\left(R P_{1}\right)$ to the first $h_{11}$ profile in $E_{3}\left(R P_{N}\right)$, a relation is proposed to increase the relative power $(R P)$ on each $f_{\text {ref }}$ :

$$
R P_{i}=R P_{1}+(i-1) \times \frac{R P_{N}-R P_{1}}{N}
$$

where $i$ is an integer that varies from 2 to $N-1 . f_{\text {ref }}$ also changes, as presented in Table 1. It passes from $300 \mathrm{~Hz}$ $\left(f_{\text {ref }}\right)$ to $150 \mathrm{~Hz}\left(f_{\text {ref }}\right)$ :

$$
f_{r e f_{i}}=f_{r e f_{1}}+(i-1) \times \frac{f_{r e f_{N}}-f_{r e f_{1}}}{N}
$$

$N$ is chosen equal to 225 . In fact, the average $f_{\text {ref }}$ is equal to $225 \mathrm{~Hz}$. In this case, 225/225 = $1 \mathrm{~s}$ needed time to switch between the impulse responses which is sufficient to consider it in continuous manner.

\section{Time-Varying $2 \times 2$ MIMO Channel}

\section{1) Relative Power Variation}

In this paragraph, we present the method used to obtain a model of a time variant channel, using the Rayleigh fading. This fading is used for the relative powers of the taps of each SISO channel. A $2 \times 2$ MIMO Rayleigh fading channel $H$ [10] is considered. It can be characterized by the relative power $P_{c}$ (resp. $P_{s}$ ) of constant (resp. scattering) channel components corresponding to LOS (resp. NLOS) paths. The ratio $P_{c} / P_{s}$ is called Ricean $K$-factor.

Assuming that all the elements of the MIMO channel matrix $H$ are Rice distributed, it can be expressed for each tap by:

$$
H=\sqrt{P_{c}} \cdot H_{F}+\sqrt{P_{s}} \cdot H_{v}
$$

where $H_{F}$ and $H_{V}$ are the constant and the scattered matrices respectively. The total received power is $P=P_{c}+P_{s}$. Thus:

$$
P_{c}=P \cdot \frac{K}{K+1}, \quad P_{s}=P \cdot \frac{1}{K+1}
$$

where $K=0$ to obtain a Rayleigh fading channel. $P$ is given in [7] for each tap. For 2 transmit and 2 receive antennas:

$$
H=\sqrt{P} \cdot\left[\begin{array}{ll}
X_{11} & X_{12} \\
X_{21} & X_{22}
\end{array}\right]
$$

To correlate the $X_{i j}$ elements, a product-based model is used. This model assumes that the correlation coefficients are independently derived at each end of the link:

$$
X=\left(R_{r}\right)^{1 / 2} \cdot H_{i i d} \cdot\left(\left(R_{t}\right)^{1 / 2}\right)^{T}
$$

$H_{i i d}$ is a matrix of independent zero means, unit variance, complex Gaussian random variables. $R_{t}$ and $R_{r}$ are the transmit and receive correlation matrices:

$$
R_{t}=\left[\begin{array}{cc}
1 & \alpha \\
\alpha^{*} & 1
\end{array}\right], \quad R_{r}=\left[\begin{array}{cc}
1 & \beta \\
\beta^{*} & 1
\end{array}\right]
$$

The complex correlation coefficients $\alpha$ and $\beta$ are expressed as:

$$
\rho=R_{x x}(D)+j \cdot R_{x y}(D)
$$

where $D=2 \pi d / \lambda, d=0.5 \lambda$ is the distance between two antennas, $\lambda$ is the wavelength and $R_{x x}$ and $R_{x y}$ are:

$$
\begin{aligned}
& R_{x x}(D)=\int_{-\pi}^{\pi} \cos (D \cdot \sin (\varphi)) \cdot \operatorname{PAS}(\varphi) \cdot d \varphi \\
& R_{x y}(D)=\int_{-\pi}^{\pi} \sin (D \cdot \sin (\varphi)) \cdot \operatorname{PAS}(\varphi) \cdot d \varphi
\end{aligned}
$$

The Power Angular Spectrum $(P A S)$ closely match the Laplacian distribution:

$$
\operatorname{PAS}(\theta)=\frac{1}{\sqrt{2} \sigma} e^{-|\sqrt{2} \theta / \sigma|}
$$

where $\sigma$ is the standard deviation of the PAS. 


\section{2) Tap Delays Variation}

The delays variation depends on the Doppler spread and on the movement direction. For $E_{1}$ and $E_{2}$, the environment is a highway (open outdoor). The delays of the multipath propagation waves vary in a linear way. They decrease if the vehicle approaches from the base station and increase if it moves away. $E_{3}$ is an urban environment. The multipath waves vary randomly when moving between the buildings and their delays depend on the movement directions. Therefore, the delays of the multipath that come from behind the vehicle (in Fig. 1) increase, while the ones from the front decrease.

\section{ARCHITECTURE AND IMPLEMENTATION}

Four FIR filters are considered to simulate the $2 \times 2$ MIMO channel. In general, the FIR width and the number of used multipliers are determined by the taps of each SISO channel. However, by simulating a scenario, the entire SISO channels have to be considered. To use the limited number of multipliers on the FPGA and to switch from one environment to another, a solution is proposed to control the change of delays by connecting each multiplier to the corresponding cells of the shift register and the memory (RAM). Therefore, the number of multipliers in the FIR filters is equal to the maximum number of taps of all SISO channels, for all environments. Thus, 4 FIR filters, each one with 9 multipliers are considered. Fig. 2 presents a FIR filter 250 with 9 multipliers (9 taps) for one SISO channel which corresponds to $E_{3}$. We have developed our own FIR filter instead of using Xilinx MAC filter to make it possible to reload the filter coefficients.

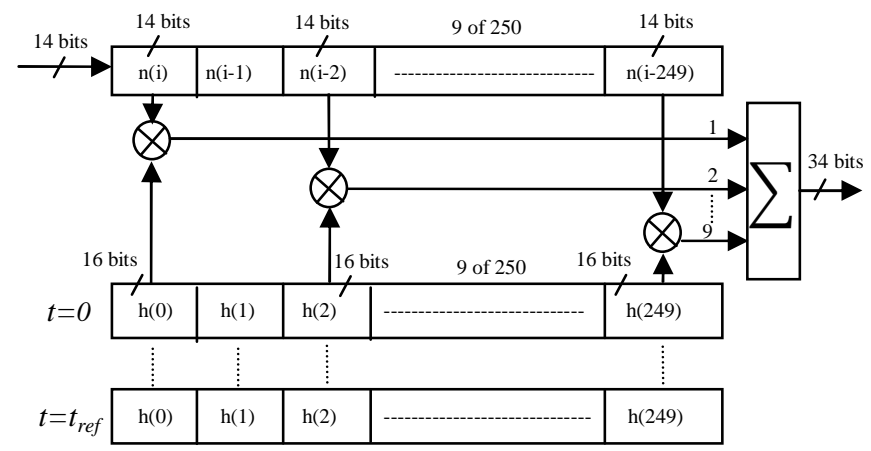

Figure 2. FIR 250 with 9 multipliers for one SISO channel using $h_{11}$.

Due to the use of a 14-bit digital-to-analog converter (DAC), the final output must be truncated. The best solution is the sliding window truncation presented in Fig. 3 which uses the 14 most significant bits.

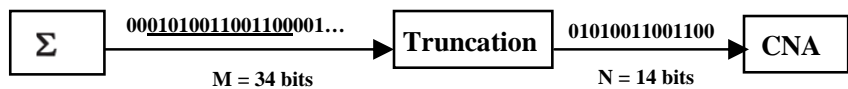

Figure 3. Sliding window truncation, from 34 to 14 bits.

Fig. 4 shows the XtremeDSP Virtex-IV board from Xilinx [2] used for the implementation of the architecture. This prototyping board is described in [14]. The simulations are made with ISE [2] and ModelSim software [17].

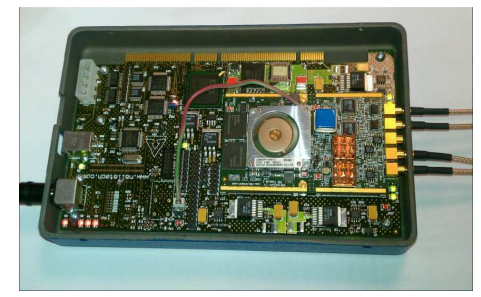

Figure 4. XtremeDSP Development board Kit-IV for Virtex-IV.

Table II shows the device utilization in one V4-SX35 for four SISO channels using four FIR filters of 9 multipliers each.

TABLE II. FPGA OCCUPATION FOR 4 FIR FILTERS

\begin{tabular}{|l|c|c|c|}
\hline Logic Utilization & Used & Available & Utilization \\
\hline Slice Flip Flops & 1,190 & 30,720 & $4 \%$ \\
\hline 4 input LUTs & 2,284 & 30,720 & $8 \%$ \\
\hline Occupied Slices & 1,805 & 15,360 & $12 \%$ \\
\hline Bonded IOBs & 40 & 448 & $9 \%$ \\
\hline BUFG/BUFGCTRLs & 1 & 32 & $4 \%$ \\
\hline FIFO16/RAMB16s & 36 & 192 & $19 \%$ \\
\hline DSP48s & 36 & 192 & $19 \%$ \\
\hline
\end{tabular}

For a MIMO profile, the sent data is: $9 \times 4=36$ words of 16 bits $=72$ bytes to transmit. Thus, the transfer speed is $72 \times f_{\text {ref }}$ $\mathrm{Bps}$, i.e. $32.4 \mathrm{kBps}$ for $\mathrm{E} 1,21.6 \mathrm{kBps}$ for $E_{2}$ and $10.8 \mathrm{kBps}$ for $E_{3}$.

\section{ACCURACY}

In order to determine the accuracy of the digital block, a comparison is made between the theoretical output signal and the Xilinx output signal. An input Gaussian signal $x(t)$ is considered and long enough to be used in streaming mode:

$$
x(t)=x_{m} e^{-\frac{\left(t-m_{x}\right)^{2}}{2 \sigma^{2}}}, \quad 0 \leq t \leq W_{t}
$$

where $W_{t}=384 \mathrm{~T}_{\mathrm{s}}, m_{x}=3 . W_{t} / 4$ and $\sigma=m_{x} / 32$ (small enough to show the effect of each path of the impulse responses on the output signals). The A/D and D/A converters of the development board have a full scale $\left[-V_{m}, V_{m}\right]$, with $V_{m}=1 \mathrm{~V}$. For the simulations we consider $x_{m}=V_{m} / 4$. The theoretic output signals are calculated by:

$$
\begin{aligned}
& y_{1}(t)=\sum_{k=1}^{9} h_{11}\left(i_{k}\right) \cdot x\left(t-i_{k} t_{s}\right)+\sum_{k=1}^{9} h_{21}\left(j_{k}\right) \cdot x\left(t-j_{k} t_{s}\right) \\
& y_{2}(t)=\sum_{k=1}^{9} h_{12}\left(p_{k}\right) \cdot x\left(t-p_{k} t_{s}\right)+\sum_{k=1}^{9} h_{22}\left(l_{k}\right) \cdot x\left(t-l_{k} t_{s}\right)
\end{aligned}
$$

The relative error is computed for each output sample by:

$$
\varepsilon(i)=\frac{Y_{\text {xilinx }}(i)-Y_{\text {theory }}(i)}{Y_{\text {theory }}(i)} .100[\%]
$$

where $Y_{\text {Xilinx }}$ and $Y_{\text {theory }}$ are vectors containing the samples of corresponding signals. The Signal-to-Noise Ratio (SNR) is:

$$
S N R(i)=20 \cdot \log _{10}\left|\frac{Y_{\text {theory }}(i)}{Y_{\text {xilinx }}(i)-Y_{\text {theory }}(i)}\right|[d B], i=\overline{1,4 N}
$$


Fig. 5 shows a snapshot of the Xilinx output signal $y_{l}$ with its relative error and $\mathrm{SNR}$ for $E_{1}, E_{2}$ and $E_{3}$ using the brutal truncation (B.T.) and the sliding truncation (S.T.).
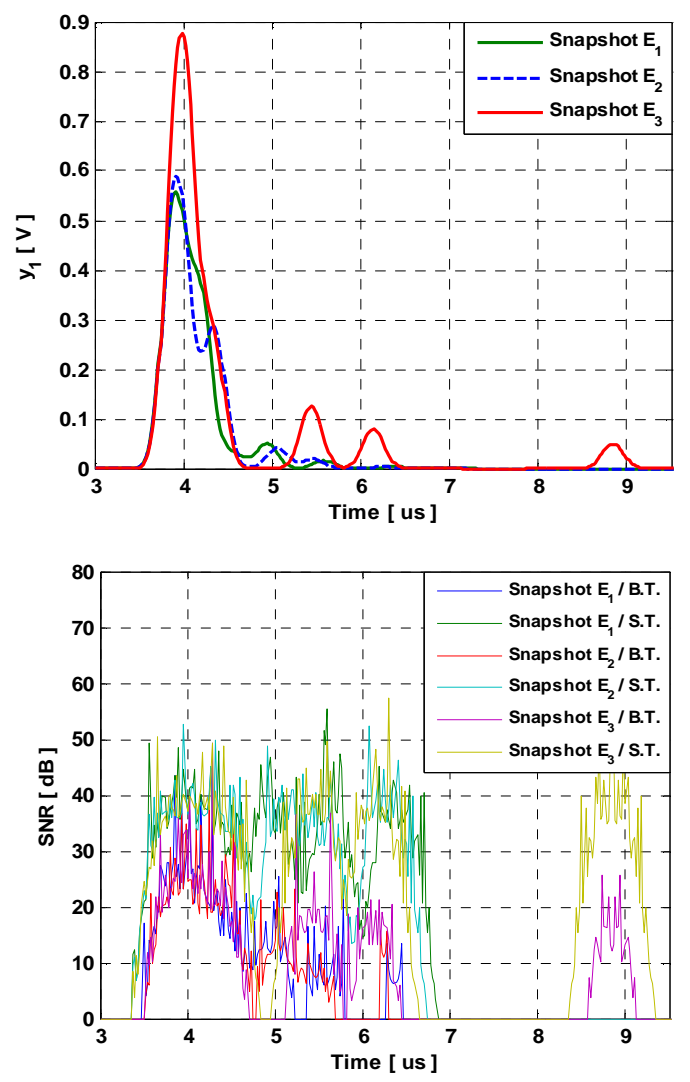

Figure 5. Snapshot of $y_{l}$ and its SNR.

The relative error is high only for small values of the output signal because the Gaussian signal test is close to 0 . The global values of the relative error and the SNR of the output signals are necessary to evaluate the accuracy of the architecture. The global relative error and SNR are computed by:

$$
\varepsilon=\frac{\|E\|}{\left\|Y_{\text {theory }}\right\|} \times 100[\%], \quad S N R_{g}=20 \times \log _{10} \frac{\left\|Y_{\text {theory }}\right\|}{\|E\|}[d B]
$$

where $E=Y_{\text {Xilinx }}-Y_{\text {theory }}$ is the error vector. Table III shows the mean global SNR for each environment.

TABLE III. MEAN GLOBAL SNR

\begin{tabular}{|c|c|c|c|c|}
\cline { 2 - 5 } \multicolumn{1}{c|}{} & \multicolumn{2}{c|}{$\boldsymbol{S N R}(\boldsymbol{d B}): \boldsymbol{y}_{\boldsymbol{1}}$} & \multicolumn{2}{c|}{$\boldsymbol{S N R}(\boldsymbol{d B}): \boldsymbol{y}_{\mathbf{2}}$} \\
\hline Environment & B.T. & S.T. & B.T. & S.T. \\
\hline$E_{1}$ & 39.23 & 74.73 & 36.95 & 73.79 \\
\hline$E_{2}$ & 40.01 & 75.22 & 37.31 & 73.66 \\
\hline$E_{3}$ & 40.09 & 78.91 & 39.37 & 75.84 \\
\hline
\end{tabular}

\section{CONCLUSION}

In this paper, tests have been made for a scenario model that switches between outdoor environments to simulate heterogeneous propagation environments. In this context, an algorithm has been proposed to switch between the environments in a continuous manner. In order to simulate a time-varying channel, a Rayleigh fading method is used to vary the relative power of the taps of the channel impulse response. Moreover, the delays variation of the taps, which depends on the Doppler spread and the proposed environment, have been considered. A solution has been proposed to control the change of delays in the architecture.

For our future work, simulations made using a Virtex-VII [2] platform will allow us to simulate up to $16 \times 16 \mathrm{MIMO}$ systems. Measurement campaigns will be carried out with the channel sounder realized by IETR, for various types of environments. The objective of these measurements is to obtain realistic MIMO channel models in order to supply the hardware simulator. A graphical user interface will also be designed to allow the user to reconfigure the simulator parameters.

\section{ACKNOWLEDGMENT}

The authors would like to thank "Région Bretagne" for its support of this work, which is a part of PALMYRE-II project.

\section{REFERENCES}

[1] S. Behbahani, R. Merched and A. Eltawil, "Optimizations of a MIMO relay network", IEEE Trans. on Signal Processing, vol. 56, no. 10, pp. 5062-5073, Oct. 2008.

[2] "Xilinx: FPGA, CPLD and EPP solutions", www.xilinx.com.

[3] P. Murphy, F. Lou, A. Sabharwal and P. Frantz, "An FPGA based rapid prototyping platform for MIMO systems", Asilomar Conf. on Signals, Systems and Computers, ACSSC, vol. 1, pp. 900-904, 9-12 Nov. 2003.

[4] Wireless Channel Emulator, Spirent Communications, 2006.

[5] Baseband Fading Simulator ABFS, Reduced costs through baseband simulation, Rohde \& Schwarz, 1999.

[6] V. Erceg, L. Shumacher, P. Kyritsi, et al., "TGn Channel Models", IEEE 802.11- 03/940r4, May 10, 2004.

[7] Agilent Technologies, "Advanced design system - LTE channel model R4-070872 3GPP TR 36.803 v0.3.0”, 2008.

[8] H. Farhat, R. Cosquer, G. Grunfelder, L. Le Coq and G. El Zein, "A dual band MIMO channel sounder at 2.2 and $3.5 \mathrm{GHz}$ ", IMTC, Victoria, BC, Canada, May 2008.

[9] P. Almers, E. Bonek et al., "Survery of channel and Radio propagation models for wireless MIMO systems", EURASIP Journal on Wireless Communications and Networking, Article ID 19070, 2007.

[10] L. Schumacher, K. I. Pedersen and P.E. Mogensen, "From antenna spacings to theoretical capacities - guidelines for simulating MIMO systems", in Proc. PIMRC Conf., vol. 2, pp. 587-592, Sep. 2002.

[11] S. Picol, G. Zaharia, D. Houzet and G. El Zein, "Hardware simulator for MIMO radio channels: design and features of the digital block", IEEE VTC Fall, Calgary, Canada, Sep. 2008.

[12] S. FouladiFard, A. Alimohammad, B. Cockburn and C. Schlegel, "A single FPGA filter-based multipath fading emulator", Globecom, Honolulu, Nov. 2009.

[13] H. Eslami, S.V. Tran and A.M. Eltawil, "Design and implementation of a scalable channel Emulator for wideband MIMO systems", IEEE Trans. on Vehicular Technology, vol. 58, no. 9, pp. 4698-4708, Nov. 2009.

[14] B. Habib, G. Zaharia and G. El Zein, "MIMO hardware simulator: new digital block design in frequency domain for streaming signals", Journal of Wireless Networking and Comm., vol. 2, no. 4, pp. 55-65, 2012.

[15] B. Habib, G. Zaharia and G. El Zein, "MIMO hardware simulator: digital block design for 802.11ac applications with TGn channel model test", IEEE VTC Spring, Yokohama, Japan, May, 2012.

[16] B. Habib, G. Zaharia and G. El Zein, "Digital block design of MIMO hardware simulator for LTE applications", ICC, Ottawa, Canada, 2012.

[17] ModelSim - Advanced Simulation and Debugging, http://model.com. 